\title{
Kernos
}

Revue internationale et pluridisciplinaire de religion grecque antique

1 | 1988

Varia

\section{L'étude monnographique des sanctuaires grecs et la réalité vécue des cultes antiques}

\section{Guy Donnay}

\section{(2) OpenEdition \\ Journals}

\section{Édition électronique}

URL : http://journals.openedition.org/kernos/101

DOI : 10.4000/kernos.101

ISSN : 2034-7871

\section{Éditeur}

Centre international d'étude de la religion grecque antique

\section{Édition imprimée}

Date de publication : 1 janvier 1988

ISSN : 0776-3824

\section{Référence électronique}

Guy Donnay, «L'étude monnographique des sanctuaires grecs et la réalité vécue des cultes antiques », Kernos [En ligne], 1 | 1988, mis en ligne le 31 janvier 2011, consulté le 30 avril 2019. URL http://journals.openedition.org/kernos/101; DOI : 10.4000/kernos.101 
Kernos, 1(1988), p. 121-127.

\section{L'ÉTUDE MONOGRAPHIQUE DES SANCTUAIRES \\ GRECS ET LA RÉALITE VÉCUE DES CULTES ANTIQUES}

Dans le cadre de ce colloque consacré à la pluridisciplinarité dans l'étude de la religion grecque antique, je voudrais attirer l'attention sur une approche des faits religieux qui me semble particulièrement nécessaire et féconde et que j'appellerai l'étude monographique des sanctuaires pour la distinguer à la fois des grandes synthèses et des monographies thématiques sur tel dieu, tel héros ou tel type de culte. Je prendrai pour guide deux ouvrages que je considère comme exemplaires, la thèse de Philippe Bruneau sur les cultes de Délos aux époques hellénistique et romaine, publiée en 1970, et celle, récente, de Madeleine Jost sur les cultes arcadiens ${ }^{1}$. En effet, l'un et l'autre se fondent sur une démarche explicite et cohérente, dont je n'ai pas jusqu'à présent trouvé l'équivalent dans d'autres publications. Certes, nous avons affaire ici chaque fois à l'oeuvre d'un seul auteur, mais nous verrons que leur démarche n'en est pas moins, et par essence, pluridisciplinaire.

Bien que beaucoup s'en défendent, les historiens de la religion grecque antique demeurent aujourd'hui encore largement tributaires des orientations définies à la fin du siècle dernier par l'école de Cambridge. Nul ne peut nier que les travaux de James George Frazer, Jane Emily Harrison et de leurs émules occidentaux, tels Erwin Rohde ou Martin P. Nilsson, ont jeté les bases scientifiques de notre discipline ${ }^{2}$ : en ramenant l'étude des religions à celle de leurs manifestations extérieures - essentiellement les mythes et les

1 Ph. BRUNEAU, Recherches sur les cultes de Délos à l'époque hellénistique et à l'époque impériale romaine, 1970; M. JOST, Sanctuaires et cultes d'Arcadie, 1985.

2 J.G. FRAZER, The Golden Bough. A Study in comparative religion, 1ère éd., 1890; J.E. HARRISON, Prolegomena to the Study of Greek Religion, 1ère éd., 1903; E. ROHDE, Psyche. Seelenkult und Unsterblichkeitsglaube der Griechen, 1893; M.P. NILSSON, Geschichte der griechischen Religion, 1ère éd., 1941. 
rites -, ils l'ont libérée de toute référence à une foi déterminée et rendue, dès lors, justiciable de méthodes objectives d'investigation, notamment l'anthropologie et l'histoire.

Ayant reconnu l'universalité du phénomène religieux à travers la diversité de ses expressions, ils furent amenés tout naturellement à privilégier l'étude comparative des mythes et des rites. Entraînés par leur enthousiasme, ils n'hésitèrent pas à se lancer d'emblée dans de vastes compilations à l'échelle de la planète tout entière, embrassant pêle-mêle des témoignages empruntés aussi bien aux auteurs antiques, grecs et latins, à la Bible hébraïque ou aux Védas indiens qu'aux enquêtes ethnographiques sur les peuples dits «primitifs» et aux traditions populaires des nations européennes.

La multiplicité et la disparate de ces témoignages ne permettaient guère une critique approfondie de chacun d'eux. Tel est d'ailleurs le principal défaut qui entache les travaux des premiers comparatistes, car, comme l'écrit justement Philippe Bruneau dans l'introduction de son livre sur les cultes de Délos en 1970,

une des faiblesses ordinaires de l'esprit, particulièrement sensible dans les travaux d'histoire religieuse antique, est d'échafauder des thèses sur des faits inexacts ou mal assurés ${ }^{3}$.

Le remède le plus sûr en l'occurrence reste la bonne vieille critique philologique et historique, prônée dès 1825 par ce pionnier trop oublié de nos études que fut Carl Otfried Müller dans ses Prolegomena zu eine wissenschaftlichen Mythologie.

Néanmoins, les efforts ultérieurs ne porteront pas tant sur l'amélioration des bases documentaires que sur la recherche de nouvelles clefs d'interprétation de faits supposés établis. Il est vrai que celles des comparatistes étaient, en général, fort sommaires et empreintes d'un positivisme quelque peu naïf. Aussi verra-t-on se développer, par réaction, des écoles interprétatives plus exigeantes, sociologique à la suite du Français Emile Durkheim, psychanalytique à la suite de l'Autrichien Sigmund Freud, phénoménologique

3 BRUNEAU, op. cit, p. 5. 
avec le Hollandais Gerardus van der Leeuw, structuraliste avec le Français Jean-Pierre Vernant, le Belge Marcel Detienne et quelques autres ${ }^{4}$.

Les structuralistes ont notamment dénoncé le danger des rapprochements «sans contrainte d'espace ni de temps ${ }^{5}$ » chers aux comparatistes et la nécessité d'analyser au préalable, de manière approfondie, chacun des témoins invoqués, pris individuellement, sous peine d'en fausser la portée et le sens et, partant, les implications qu'on en peut tirer. Mais leur objectif n'en reste pas moins, comme celui des autres écoles, de définir des catégories générales, fussent-elles assorties d'infinies nuances.

L'objectif de l'étude monographique est à la fois plus modeste et plus ambitieux. Plus modeste, car elle se limite à une période et à un territoire déterminés. Plus ambitieux, car elle prétend rassembler et traiter de manière, si possible, exhaustive tous les témoignages littéraires, épigraphiques, archéologiques, numismatiques.. - relatifs aux cultes attestés dans ce territoire à cette période. La seule énumération des sources qu'elle met en cuvre rend, par ailleurs, évident son caractère pluridisciplinaire. Philippe Bruneau l'a définie avec une clarté et une concision parfaites :

Je résumerai mes intentions en disant que j'ai voulu faire une monographie et (en dépit de la valeur péjorative que l'usage attache à ce mot) une compilation critique. [...] le but auquel j'ai tendu - sans y parvenir trop souvent, on s'en doute - était de rendre compte de tous les documents relatifs, de près ou de loin, à l'histoire dẹs cultes déliens ${ }^{6}$.

La compilation critique porte à la fois sur les sources et sur les interprétations qui en ont été données à des époques et dans des contextes divers. En effet, peu de témoignages antiques sont suffisamment clairs et complets pour se passer d'exégèse, et cela est vrai

4 E. DURKHEIM, Les formes élémentaires de la vie religieuse, 1912; S. FREUD, Totem und Tabu, 1913; G. VAN DER LEEUW, Phänomenologie der Religion, 1933. Pour le structuralisme, il n'existe pas d'ouvrage général auquel on puisse se référer, mais seulement des études particulières, trop nombreuses pour les citer ici.

5 M. DETIENNE, Dionysos mis à mort, 1976, p. 26.

6 BRUNEAU, op. cit., p. 6. 
davantage encore de la documentation archéologique. Car «le témoignage des documents - surtout des documents figurés - ne se laisse pas entendre dès l'abord et l'on doit le solliciter ${ }^{7}$.

Dans ce dernier domaine, qui relève de l'archéologie, la critique ne sera pertinente et efficace que si l'auteur a une connaissance des documents. Ici encore, nos deux guides s'avèrent exemplaires : Philippe Bruneau a rédigé sa thèse, pour ainsi dire, dans les ruines mêmes de l'île sainte d'Apollọn et Madeleine Jost a poussé le scrupule jusqu'à visiter la plupart des sites de l'Arcadie antique, malgré leur accès souvent malaisé, et à examiner personnellement les vestiges qui en proviennent, bien qu'ils soient disséminés dans différents dépôts.

L'importance du contrôle direct vaut aussi pour les inscriptions, dont on sait qu'un œil plus ou moins exercé ne les déchiffre pas toujours de la même façon et dont bien souvent une meilleure connaissance du contexte permet des lectures plus précises. Quant aux textes littéraires, la règle d'or reste de les traduire soi-même et ce quelle que soit la qualité des traductions existantes, la traduction étant, comme chacun sait, le premier des commentaires. Est-il nécessaire d'ajouter que nos deux auteurs se sont imposé l'un et l'autre cette salutaire discipline?

Une fois le contenu des témoignages établi et vérifié, «les diverses catégories de documents doivent, comme l'écrit Madeleine Jost, être confrontées et mises à l'épreuve les unes des autres si l'on veut essayer d'établir les faits dans leur authenticité, puis, si c'est possible, d'en tracer la genèse et de leur donner un sens ${ }^{8}$ ». C'est ici qu'intervient, inévitablement, la subjectivité du chercheur. Mais celui-ci prouvera son honnêteté en mettant à la disposition du lecteur la totalité de la documentation sur laquelle il se fonde, lui offrant ainsi «de vérifier ses assertions, de les compléter et, tout autant, de les rejeter ${ }^{9} »$.

\footnotetext{
7 BRUNEAU, op. cit., p. 6.

8 JOST, op. cit., p. 6.

9 BRUNEAU, op. cit., p. 5.
} 
L'esprit critique qu'il suppose ainsi à son lecteur, l'auteur se doit de l'exercer d'abord lui-même sous peine de faire cuvre, non de savant, mais de simple documentaliste. La profession de foi de Philippe Bruneau est, à cet égard, éclairante :

Je me suis attaché à ne rien croire avant d'avoir suivi moi-même la démarche des sources aux conclusions; il m'est même arrivé de développer les raisons d'accepter un fait parfaitement admis par mes devanciers, parce qu'il me paraissait que l'affirmation n'allait pas de soi et qu'il importait de tenter la démonstration. Souvent aussi, j'ai amplement taillé dans la forêt des conjectures et me tiendrai pour satisfait si j'ai pu faire mesurer par mon lecteur le degré de certitude ou d'incertitude auquel atteint telle démonstration ou telle hypothèse ${ }^{10}$.

Dans l'interprétation, déclare, de son côté, Madeleine Jost, je ne me référerai pas à un système de «décodage» unique [...]. Analyse et comparaison seront mes deux armes essentielles. Dans l'analyse, je m'efforcerai de ne pas trop détruire l'unité des données : les faits constituent, malgré tout, un ensemble et l'analyse ne doit pas perdre de vue leur signification globale. [...] Pour les comparaisons, [...] j'éviterai de tomber dans le piège «comparaison-assimilation» qui annihile toute spécificité. Enfin, je m'en tiendrai obstinément aux faits, plus soucieuse d'ébranler les fausses certitudes lorsqu'elles sont mal fondées que de multiplier les hypothèses : les apories valent aussi d'être soulignées ${ }^{11}$.

En particulier, et au risque d'être traitée de traditionalisme, l'étude monographique doit se garder des interprétations orientées vers la confirmation de théories préexistantes. Dans la négative, elle perdrait toute originalité et, ce qui est plus grave, toute possibilité de remettre en question les idées reçues. Or, cette remise en question est sa véritable raison d'être, puisque son premier objectif est d'établir la réalité ou le degré de vraisemblance des faits religieux attestés dans le cadre géographique et chronologique choisi afin de permettre la construction ultérieure d'éventuelles synthèses sur des bases saines.

Un autre intérêt - sinon le principal avantage - de l'étude monographique est de replacer les faits religieux dans leur réalité vécue. L'obligation d'exhaustivité lui impose, en effet, de ne négliger aucun aspect ni aucun fait, aussi secondaire pût-il apparaître au premier abord. Philippe Bruneau et Madeleine Jost ont ainsi sorti de

10 Ibid.

11 JOST, op. cit., p. 6-7. 
l'ombre mille détails relatifs à l'organisation des cultes, à la construction et à l'entretien des sanctuaires publics ou privés, au recrutement de leur desservants. De cette masse d'informations ponctuelles se·dégagent quelques impressions d'ensemble grâce auxquelles nous pouvons mieux appréhender l'esprit dans lequel ces cultes étaient pratiqués. La piété de l'homme de la rue ne s'adressaitelle pas davantage aux innombrables héros et petits dieux locaux ou aux hypostases spécialisées des grandes divinités - parmi lesquelles

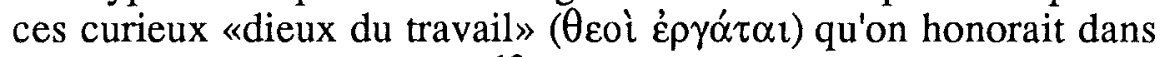
un faubourg de Mégalopolis ${ }^{12}$ - plutôt qu'aux vedettes du panthéon chantées par les poètes?

La connaissance de la réalité vécue des cultes antiques fournit aussi des explications simples et pertinentes à des faits dont on serait tenté de tirer abusivement des conséquences théoriques. Ainsi, par exemple, Philippe Bruneau a montré que, à Délos, le cumul occasionnel du canéphorat de Dionysos avec la prêtrise d'Artémis résultait tout simplement du recrutement des titulaires de ces fonctions dans un groupe relativement limité, celui des jeunes filles de bonne famille : il ne prouve nullement l'existence de liens privilégiés entre les deux divinités ${ }^{13}$. En revanche, dans cette île cosmopolite, Héraklès apparaît bien, aux époques hellénistique et romaine, comme une sorte de divinité syncrétique dans laquelle chaque peuple retrouvait un des siens, les Grecs le dieu du gymnase, les Italiens leur Hercule, les Tyriens leur Melqart, les Palestiniens de Jabné leur Baal $^{14}$.

L'étude monographique nous met enfin en garde contre la tentation de considérer les faits religieux attestés par les textes et les monuments comme devant nécessairement s'insérer dans une organisation structurée de croyances et de pratiques, la religion grecque. Au contraire, elle souligne la diversité des particularismes locaux et les aspects divers qu'une même divinité - ou des divinités portant le même nom ? - peut prendre selon le lieu où elle est honorée. Dans cette perspective, la question que se pose Madeleine Jost interpelle notre réflexion :

12 JOST, op. cit., p. 232-233 et 452.

13 BRUNEAU, op. cit., p. 201.

14 BRUNEAU, op. cit., p. 411-412. 
Et si la religion grecque elle-même, au lieu d'être un ensemble bien organisé dont il faut saisir la cohérence, n'était qu'un agrégat de pratiques accumulées au cours des temps $?^{15}$

Musée Royal de Mariemont

Guy DONNAY

B - 6510 MORLANWELZ

15 JOST, op. cit., p. 6. 